Psychother Psychosom 2016;85:117-118

DOI: $10.1159 / 000441282$

\section{Working Alliance in Internet-Based Cognitive- Behavioral Therapy for Obsessive-Compulsive Disorder}

\author{
Nirmal Herbst ${ }^{\mathrm{a}}$, Guido Franzen ${ }^{\mathrm{a}}$, Ulrich Voderholzer ${ }^{\mathrm{a}, \mathrm{b}}$, \\ Nicola Thiela , Christine Knaevelsrud', Elisabeth Hertenstein ${ }^{a}$, \\ Christoph Nissen a , Anne Katrin Külz ${ }^{\mathrm{a}}$, \\ a Department of Psychiatry and Psychotherapy, University \\ Medical Center Freiburg, Freiburg, 'b Schoen Clinic Roseneck, \\ Prien, and ${ }^{\mathrm{C}}$ Department of Clinical Psychology and \\ Psychotherapy, Free University Berlin, Berlin, Germany
}

The patient-therapist relation has consistently been found to be of importance for the outcome of psychotherapy with an overall effect size of $r=0.275$ [1]. The same relevance of the patient-therapist relation holds true for the treatment of obsessive-compulsive disorder (OCD) [2]. Cognitive-behavioral psychotherapy (CBT) with exposure and response prevention (ERP) constitutes an effective treatment for OCD and is recommended as the first-line treatment [3]. Yet, only a minority of patients receives it [4]. The developing field of Internet-based CBT (iCBT) [5] is a promising opportunity for further dissemination. Due to the anxiety-provoking nature of ERP, the treatment is associated with high levels of discomfort and is often perceived as demanding. Consequently, it seems of particular importance to establish a stable patient-therapist relation.

While the findings on the patient-therapist relation mentioned above apply to face-to-face psychotherapy, less is known about its relevance and potential differences regarding the characteristics of iCBT (e.g. in many cases text-based and asynchronous communication, lack of nonverbal cues). A systematic review found no differences in the patient-therapist relation between iCBT and faceto-face therapy [6]. The short form of the Working Alliance Inventory (WAI-SR) [7] scored in two samples at 72 and $84 \%$ of the maximum scale value, respectively [7]. So far, only one study of iCBT for OCD reported on the patient-therapist relation [8]. Even though the authors found a high working alliance, the result may not be generalizable to other - especially text-based - iCBT approaches for OCD due to some major limitations (only 6 participants, phone- or videoconference-based treatment).

Furthermore, it is important to identify factors predicting the development of the patient-therapist relation. Results on the faceto-face treatment of OCD showed a negative correlation between pretreatment symptom severity and the development of the patient-therapist relation [9]. For iCBT, the review by Sucala et al. [6] pointed towards this factor and the communication modality to be of influence on the patient-therapist relation.

The present data analysis was part of a randomized controlled trial on the efficacy of a text-based iCBT with therapist guidance and ERP for treating patients with OCD. In this trial, we observed a high level of acceptance and strong and stable effect sizes of the intervention [10]. The present analysis focused on the patienttherapist relation. Thirty participants with a primary OCD diagnosis according to ICD-10 criteria completed the iCBT intervention (14 sessions) and the posttreatment outcome measures. The WAI-SR was used to assess the patient-therapist relation. Obsessive-compulsive symptoms were assessed using the Yale-Brown Obsessive Compulsive Scale Self-Report (Y-BOCS SR) and the Obsessive-Compulsive Inventory - Revised (OCI-R). For data analysis, mean composite scores, change scores, Spearman's rank correlations and a multiple regression model were calculated.

With an arithmetical mean of $4.08(\mathrm{SD}=0.78)$, the posttreatment WAI-SR composite score represents $77 \%$ of the maximum scale value, which indicates a high working alliance. The mean scores for the subscales are as follows: task $=3.82(\mathrm{SD}=0.86)$, goal $=4.11(\mathrm{SD}=0.94)$, bond $=4.32(\mathrm{SD}=0.78)$. The patienttherapist relation was significantly correlated with the Y-BOCS SR change score $(\mathrm{r}=0.33, \mathrm{p}=0.04$; Y-BOCS SR: before $=19.87$, $\mathrm{SD}=5.92$; after $=14.63, \mathrm{SD}=6.28)$. A marginal significant correlation was found between the OCI-R change score and the WAI-SR $(\mathrm{r}=0.29, \mathrm{p}=0.06$; OCI-R: before $=24.77, \mathrm{SD}=9.97$; after $=16.57$, $\mathrm{SD}=9.69)$. Relevant parameters for all included variables of the linear multiple regression model $(n=29)$ are listed in table 1 .

Together, the results provide evidence that a solely text-based iCBT for OCD does not hinder a high working alliance. On the contrary, a strong and stable patient-therapist relation could be established, which was comparable to prior results for traditional face-to-face treatment (see above). Without any verbal or visual interaction between patients and therapists, the three dimensions of the working alliance, goal, task and bond, were implemented. With regard to the management of potential adverse events, including suicidal ideation, the therapeutic bond seems especially important when having no direct personal contact as in iCBT, since a high level of trust may promote patients confiding in their therapist about delicate issues, thereby allowing for a prompt response.

The observed correlation with the reduction of OCD symptoms suggests that fostering the working alliance is important in iCBT just as it is in face-to-face treatment. However, as always with correlations, the causality is not clear. Since the working alliance

Data presented in this article were taken from the unpublished master's thesis of Guido Franzen at the University of Freiburg.

\section{KARGER}

E-Mail karger@karger.com

www.karger.com/pps (c) 2016 S. Karger AG, Basel

0033-3190/16/0852-0117\$39.50/0
Dr. Nirmal Herbst, Dipl.-Psych.

Department of Psychiatry and Psychotherapy University Medical Center Freiburg

Hauptstrasse 5, DE-79104 Freiburg (Germany)

E-Mail Nirmal.Herbst@arcor.de 
Table 1. Summary of the multiple regression analysis to predict the posttreatment patient-therapist relation of the WAI-SR using pretreatment symptom measures $(\mathrm{n}=29)$

\begin{tabular}{lrrrrl}
\hline Model & B & SE B & $\beta$ & $\mathrm{t}$ & $\mathrm{p}$ \\
\hline Constant & 2.803 & 1.437 & & 1.950 & 0.069 \\
Age & 0.049 & 0.023 & 0.606 & 2.081 & $0.054^{*}$ \\
Gender & 0.633 & 0.470 & 0.399 & 1.348 & 0.196 \\
Living situation & 0.046 & 0.242 & 0.049 & 0.191 & 0.851 \\
Duration of OCD & -0.036 & 0.023 & -0.463 & -1.549 & 0.141 \\
OCI-R subscale & & & & & \\
$\quad$ Washing & 0.028 & 0.061 & 0.147 & 0.459 & 0.652 \\
$\quad$ Obsessing & -0.072 & 0.089 & -0.278 & -0.812 & 0.429 \\
$\quad$ Hoarding & -0.035 & 0.062 & -0.144 & -0.556 & 0.586 \\
$\quad$ Ordering & -0.115 & 0.053 & -0.539 & -2.179 & $0.045^{* *}$ \\
$\quad$ Checking & 0.033 & 0.064 & 0.154 & 0.519 & 0.611 \\
$\quad$ Neutralizing & 0.171 & 0.088 & 0.688 & 1.933 & $0.071^{*}$ \\
Y-BOCS SR subscale & & & & & \\
$\quad$ Obsessions & -0.090 & 0.082 & -0.439 & -1.103 & 0.286 \\
$\quad$ Compulsions & -0.018 & 0.071 & -0.078 & -0.253 & 0.804 \\
\hline
\end{tabular}

$\mathrm{R}^{2}=0.710$, adjusted $\mathrm{R}^{2}=0.133, \mathrm{p}=0.279$, list-wise deletion of missing values. ${ }^{* *} \alpha<0.05$ : significant contribution to the model; $* a<0.10$ : marginal significant contribution to the model.

was only assessed after treatment, it cannot be ruled out that patients with a greater symptom reduction gave their working alliance a better rating in hindsight.

In contrast to our expectations, the severity of initial OCD symptoms had no significant predictive value for the development of the working alliance. This indicates that an iCBT approach works not only for patients with mild OCD symptoms, but also for those with a higher symptom severity, as they too can develop a high working alliance and subsequently have the opportunity to profit from the treatment. Regarding subtypes of OCD, further studies with larger samples are needed to draw reliable conclusions. However, the significant and marginally significant results (table 1) may indicate that younger patients with a specific neutralizing compulsion - like washing or checking - are more likely to develop a stable and good working alliance than patients suffering from an ordering subtype of OCD.

Some limitations restrict the generalizability of the present findings. The sample size was relatively small and the WAI-SR was only assessed once after treatment and only for patients, not for therapists. Based on our findings, future investigations with a larger sample could realize detailed subgroup analyses to determine which patient characteristics are linked to developing a higher working alliance. Overall, the development of a strong and stable working alliance in text-based iCBT for OCD is an important find- ing to open the path for further implementation of this method allowing for a broader dissemination of CBT as the first-line treatment for OCD. However, it should be noted that this access to treatment cannot be seen as a substitute for face-to-face treatment in general but offers an alternative with its own strengths and weaknesses.

\section{Disclosure Statement}

Funding for this study was provided by the German Research Foundation (DFG), grant KU 2754/1-1. The DFG had no role in the study design, collection, analysis or interpretation of the data, preparation, review or approval of the manuscript, or the decision to submit the paper for publication. The authors are not aware of any affiliations, memberships, funding, or financial holdings that might be perceived as affecting the objectivity of this work.

\section{Statement of Ethics}

The study was approved by the local ethics committee of the University Medical Center in Freiburg/Germany and is registered in the German Clinical Trials Register (Main ID: DRKS00004612).

\section{References}

1 Horvath AO, Del Re AC, Flückiger C, Symonds D: Alliance in individual psychotherapy. Psychotherapy 2011;48:9-16.

2 Vogel PA, Hansen B, Stiles TC, Götestam KG: Treatment motivation, treatment expectancy, and helping alliance as predictors of outcome in cognitive behavioral treatment of OCD. J Behav Ther Exp Psychiatry 2006:37:247-255.

3 NICE: Obsessive-Compulsive Disorder: Core Interventions in the Treatment of Obsessive-Compulsive Disorder and Body Dysmorphic Disorder. Leicester/London, British Psychological Society/Royal College of Psychiatrists, 2006.

4 Schwartz C, Schlegl S, Kuelz AK, Voderholzer U: Treatment-seeking in OCD community cases and psychological treatment actually provided to treatment-seeking patients: a systematic review. J Obsess Compuls Relat Disord 2013;2:448-456.

5 Bee P, Bower P, Lovell K, Gilbody S, Richards D, Gask L, et al: Psychotherapy mediated by remote communication technologies: a meta-analytic review. BMC Psychiatry 2008;8:60.

6 Sucala M, Schnur JB, Constantino MJ, Miller SJ, Brackman EH, Montgomery GH: The therapeutic relationship in E-therapy for mental health: a systematic review. J Med Internet Res 2012;14:e110.

7 Hatcher RL, Gillaspy JA: Development and validation of a revised short version of the working alliance inventory. Psychother Res 2006;16:1225.

8 Vogel PA, Launes G, Moen EM, Solem S, Hansen B, Haland AT, et al: Videoconference- and cell phone-based cognitive-behavioral therapy of obsessive-compulsive disorder: a case series. J Anxiety Disord 2012;26: $158-164$.

9 Eaton TT, Abeles N, Gutfreund MJ: Therapeutic alliance and outcome: impact of treatment length and pretreatment symptomatology. Psychotherapy $1988 ; 25: 536-542$.

10 Herbst N, Voderholzer U, Thiel N, Schaub R, Knaevelsrud C, Stracke S, et al: No talking, just writing! Efficacy of an Internet-based cognitive behavioral therapy with exposure and response prevention in obsessive compulsive disorder. Psychother Psychosom 2014;83:165-175. 